Communications in Physics, Vol.20, No. 1 (2010), pp. 31-36

\title{
DYNAMICS OF COLLAPSE OF OPTICAL PULSES IN KERR MEDIUM PLACED IN A HARMONIC POTENTIAL
}

\author{
CAO LONG VAN \\ Institute of Physics, University of Zielona Góra, Podgórna 50, \\ 65-246 Zielona Góra, Poland \\ DINH XUAN KHOA \\ Vinh University, Nghe An, Vietnam \\ NGUYEN VIET HUNG \\ Soltan Institute for Nuclear Studies, Hoża 69, 00-681 Warsaw, Poland \\ M. TRIPPENBACH \\ Department of physics, Warsaw University, Hoża 69, 00-681 Warsaw, Poland
}

\begin{abstract}
We consider the propagation of optical pulses in Kerr medium placed in a harmonic potential for 2D case in framework of Variational Approximation (VA). We will use two types of trial function: gaussian and hyperbolic secant. We will show that for the values of the nonlinearity parameter bigger than a certain critical value the pulses will collapse. This corresponds to the physical situation when the self-focusing dominates over the dispersion and diffraction. We will confirm this by numerical calculations which are in excellent agreement with the VA predictions.
\end{abstract}

\section{INTRODUCTION}

Consideration of the self-focusing effect in nonlinear optics is interesting both theoretically and practically. Theoretically, because sometimes we can see dramatic concurrence between different nonlinear effects of the pulse propagation in the nonlinear medium. When the self-focusing effect dominates over the other effects as the dispersion, diffraction etc. the amplitude of the optical pulse (soliton) increases drastically. In a critical point the pulse completely collapses. In practice this phenomenon is very dangerous because it usually destroys the optical material. By analogy between nonlinear optics in a Kerr medium and the Bose-Einstein condensate (BEC) system [1], all results of this consideration can be transferred in to the BEC systems. For some values of the nonlinear coupling constant we can have the collapse and the explosion of the BEC [2]. The collapse of self-focusing waves described by the nonlinear Schrödinger equation (NLSE) in nonlinear optics and plasma turbulence is reviewed in [5]. In this paper using the variational approximation (VA) we will predict the critical point in which the optical pulse collapses. In the VA the choice of the trial functions is essential. There are two perspective ones for this situation, namely the Gaussian Ansatz and the Secant Ansatz. Here we have done variational calculations for two types of such trial functions. By confirming numerically our analytical predictions through the use of time imaginary method [3] we have concluded that the secant trial function is more proper. This fact is quite understandable because 
the "non-perturbed" solution is the secant hyperbolic solution. In following section, we will show the calculations in the case secant ansatz.

\section{VARIATIONAL APPROXIMATION}

We investigate 2D-NLSE describing the propagation of the pulse in a Kerr medium placed in a harmonic potential [1]:

$$
i \Psi_{t}=-\frac{1}{2}\left(\Psi_{x x}+\Psi_{y y}\right)+\frac{\omega^{2} x^{2}}{2} \Psi-g|\Psi|^{2} \Psi
$$

where $\mathrm{g}$ is a nonliear coefficient.

To find soliton solutions of this equation, we use the following Secant Ansatz in two directions $\mathrm{x}$ and $\mathrm{y}$ :

$$
\Psi(x, y, t)=A(t) \operatorname{sech}\left(\frac{x}{W(t)}\right) \operatorname{sech}\left(\frac{y}{V(t)}\right) \exp \left\{i\left(\phi(t)+\frac{1}{2}\left(b(t) x^{2}+\beta(t) y^{2}\right)\right)\right\}
$$

Here the variational parameters $A(t), \phi(t), b(t), \beta(t), W(t)$ and $V(t)$ stand for the amplitude, total phase, spatial chirp coefficients, transverse widths along $\mathrm{x}$ and $\mathrm{y}$ directions, respectively. The Lagrange Function in our VA scheme has the form [4]:

$$
L=\frac{1}{2} \int_{-\infty}^{\infty} \int_{-\infty}^{\infty} \mathrm{d} x \mathrm{~d} y\left[i\left(\Psi_{t} \Psi^{*}-\Psi_{t}^{*} \Psi\right)-\left|\Psi_{x}\right|^{2}-\left|\Psi_{y}\right|^{2}-\omega^{2} x^{2}|\Psi|^{2}+g|\Psi|^{4}\right] .
$$

We substitute now our Ansatz into above Lagrange Function and integrate over $x$ and $y$. Consequently we obtain:

$$
L=4 A^{2} W V\left[\frac{2 g A^{2}}{9}-\phi^{\prime}-\frac{1}{6}\left(\frac{1}{W^{2}}+\frac{1}{V^{2}}\right)-\frac{\pi^{2}}{24}\left(V^{2}\left(\beta^{\prime}+\beta^{2}\right)+W^{2}\left(b^{\prime}+b^{2}+\omega^{2}\right)\right)\right] .
$$

The Euler-Lagrange equations for the parameters as the dynamical variables lead to the following system of differential equations:

$$
\begin{gathered}
b=\frac{W^{\prime}}{W} \\
\beta=\frac{V^{\prime}}{V}, \\
W^{\prime \prime}=\frac{4}{\pi^{2} W^{3}}-\frac{2 g}{3 \pi^{2} V W^{2}}-\omega^{2} W \\
V^{\prime \prime}=\frac{4}{\pi^{2} V^{3}}-\frac{2 g}{3 \pi^{2} W V^{2}}, \\
\phi^{\prime}=\frac{g}{9 W V}-\frac{1}{6}\left[\frac{1}{V^{2}}+\frac{1}{W^{2}}+\frac{\pi^{2}}{4}\left(\omega^{2} W^{2}+W W^{\prime \prime}+V V^{\prime \prime}\right)\right],
\end{gathered}
$$

and

$$
A^{2} W V=\text { const. }
$$

For our ansatz we have:

$$
\int_{-\infty}^{\infty} \int_{-\infty}^{\infty}|\Psi|^{2} \mathrm{~d} x \mathrm{~d} y=4 A^{2} W V
$$


Therefore we can conclude:

$$
4 A^{2} W V=1 .
$$

We are now looking for the stationary solution by setting $W^{\prime}=W^{\prime \prime}=0$ and $V^{\prime}=V^{\prime \prime}=0$ in these equations. Then we receive the following solutions:

$$
\begin{aligned}
\beta & =b=0, \\
V & =\frac{6}{g} W \\
W & =\sqrt[4]{\frac{36-g^{2}}{9 \pi^{2} \omega^{2}}} .
\end{aligned}
$$

The form of the last formula leads to the necessary condition for the existence of solitons: the value of nonlinearity $g$ should satisfy the inequality:

$$
g<6 .
$$

The value $g_{c}=6$ is a critical value. If the nonlinear coefficient is larger than $g_{c}$ then the collapse occurs. The pulse will be very narrow and its amplitude will go to the infinity. We confirm this result by using direct numerical time imaginary method introduced in [3]. Numerical results are displayed on Fig. $1(g=3, \omega=4)$ and Fig. $2(g=5.9, \omega=4)$.

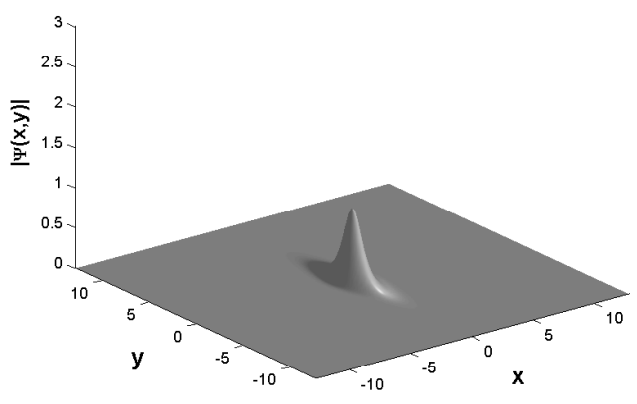

Fig. 1. Soliton solution with nonlinearity which is below critical value

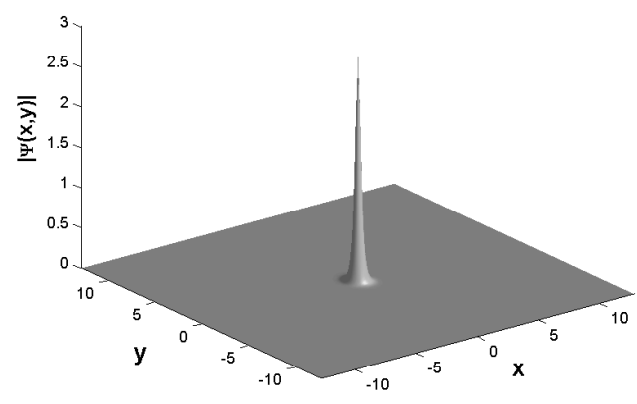

Fig. 2 Soliton solution with nonlinearity which is approximately equal to the critical value

In Fig. 2, the nonlinear coefficient is closed to the critical value. The pulse is very sharp and narrow. Its amplitude will go to infinity when the nonlinear coefficient approaches the critical value.

\section{OPTICAL CHEMICAL POTENTIAL}

In the case of the soliton solution, we can also find it in the following form:

$$
\Psi(x, y, t)=e^{-i \mu_{2 D} t} \Phi(x, y),
$$




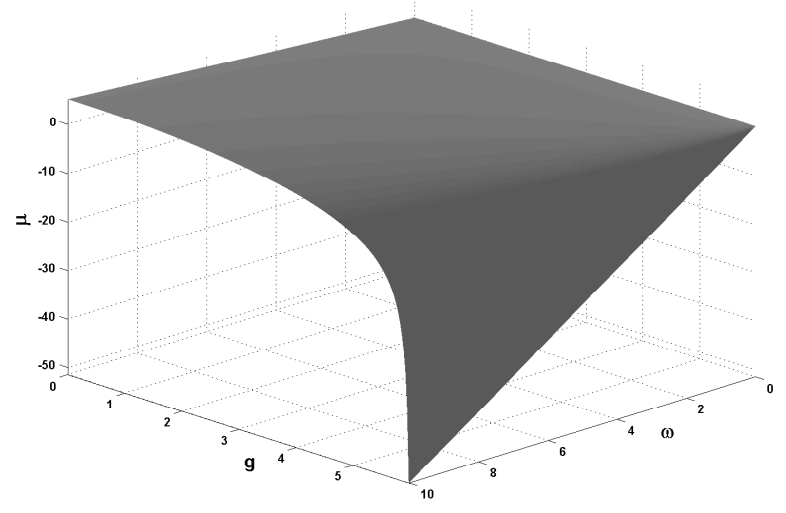

Fig. 3. Chemical potential from prediction of VA.

where $\Phi(x, y)$ is a real function and $\mu_{2 D}$ is a "optical chemical potential" (OCP) which is strictly related to the energy of the pulse.

We substitute expressions (14), (15) of the widths of the soliton with the equation (9) for $\phi^{\prime}$. Then we obtain:

$$
\phi^{\prime}=-\frac{\pi \omega}{18} \frac{18-g^{2}}{\sqrt{36-g^{2}}}
$$

Hence the phase of soliton is:

$$
\phi(t)=\int \phi^{\prime} d t=-\frac{\pi \omega t}{18} \frac{18-g^{2}}{\sqrt{36-g^{2}}} .
$$

From above definition of the optical chemical potential (17), in comparison with our ansatz we can easily conclude that:

$$
\phi(t)=-\mu_{2 D} t
$$

Therefore:

$$
\mu_{2 D}=\frac{\pi \omega}{18} \frac{18-g^{2}}{\sqrt{36-g^{2}}} .
$$

The value of OCP is displayed on Fig. 3. This quantity is a function of both variables $g$ and $\omega$.

From the result of the paper [4], we can derive expression of the OCP, which corresponds to the Gaussian Ansatz as follows:

$$
\mu_{2 D}=\frac{\omega}{2 \pi} \frac{2 \pi^{2}-g^{2}}{\sqrt{4 \pi^{2}-g^{2}}} .
$$

The critical value of nonlinearity for this case is $g_{c}=2 \pi$.

Now we again confirm predictions of VA by using numerical calculation. The values of OCP versus $g$ when value of $\omega$ is fixed $(\omega=2.5)$ are illustrated on Fig. 4. The circles are numerical results, the dashed line corresponds to the Gaussian Ansatz and the 


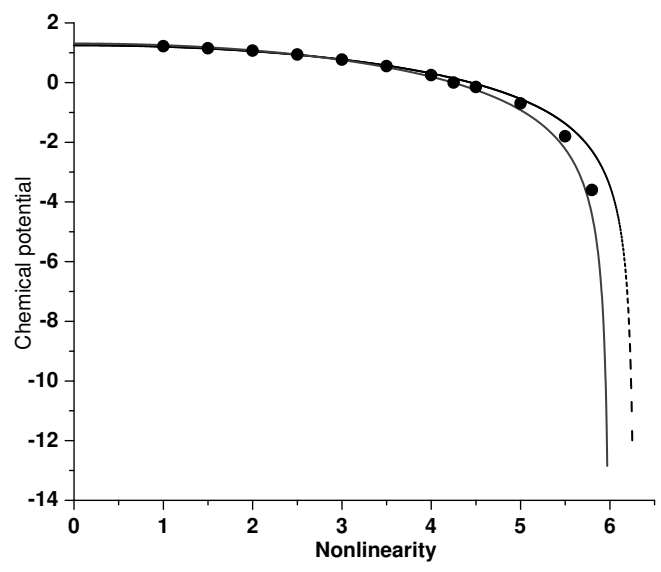

Fig. 4. Chemical potential versus nonlinearity when $\omega=2.5$.

continuous line corresponds to the Secant one. We see that the continuous line approaches the numerical results more closely, so the trial secant function is better than the gaussian one.

\section{DYNAMICS OF THE COLLAPSE}

We now consider the case in which $g \geq 6$. In this regime the collapse occurs. Then we have:

$$
V=\frac{6}{g} W \simeq W
$$

Therefore the fact of the cylindrical symmetry can be used. We can drop the harmonic term and obtain:

$$
W^{\prime \prime}=\frac{4}{\pi^{2} W^{3}}-\frac{2 g}{3 \pi^{2} W^{3}}=\frac{(12-2 g)}{3 \pi^{2} W^{3}} .
$$

We set:

$$
\lambda=\frac{2(g-6)}{3 \pi^{2}}>0 .
$$

This leads to the following ordinary differential equation for the width $W(t)$ :

$$
W^{\prime \prime}=-\frac{\lambda}{W^{3}} .
$$

We can easily solve it with the initial conditions: $W(0)=W_{0}, W^{\prime}(0)=0$. Here $W_{0}$ is a certain value of the initial width of the pulse and

$$
W^{\prime}(0)=0
$$

for stationary solution. 


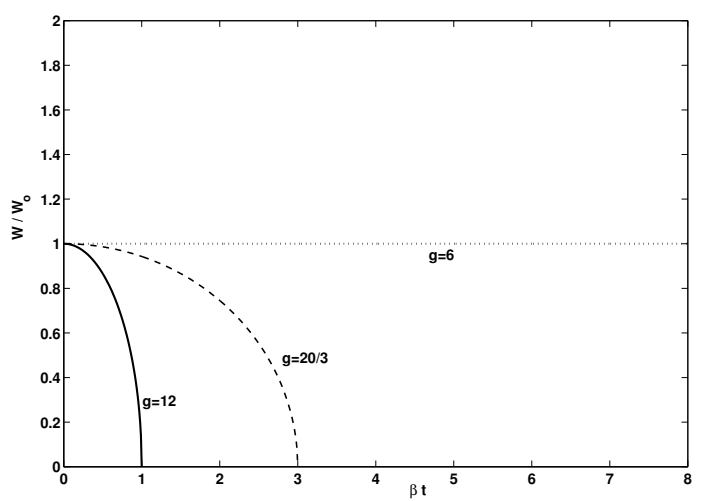

Fig. 5. Pulse width $W(t)$ normalized to $W_{0}$ versus $\beta t$ (with $\beta=\frac{2}{\pi W_{0}^{2}}$ ) for different values of $g$ in the case of collapses.

After integrating the equation (26) we obtain the solution:

$$
W(t)=W_{0} \sqrt{1-\frac{\lambda t^{2}}{W_{0}^{4}}}
$$

Now we introduce the time of collapse $t_{c o l}$ by demanding:

$$
W\left(t_{c o l}\right)=W_{0} \sqrt{1-\frac{\lambda t_{c o l}^{2}}{W_{0}^{4}}}=0 .
$$

It follows that

$$
t_{c o l}=\frac{W_{0}^{2}}{\sqrt{\lambda}}
$$

Fig. 5 shows the change of width of the pulse when the collapse occurs. We illustrated it numerically for some values of the nonlinearity. We see now that we have a similar situation as on the Fig. 2 in [5].

\section{REFERENCES}

[1] Cao Long Van, Dinh Xuan Khoa, Marek Trippenbach, Introduction to Nonlinear Optics, Vinh 2003.

[2] E. A. Donley et al. Nature 412 (2001) 295.

[3] Cao Long Van, Dinh Xuan Khoa, Nguyen Viet Hung, Marek Trippenbach, Application of the Time Imaginary Method in the Quest for Soliton Solutions, the 32th National Conference on Theoretical Physics, Da Nang, 2008.

[4] Cao Long Van, Dinh Xuan Khoa, Nguyen Viet Hung, Marek Trippenbach, Reduction of 2D Problem of Bose - Einstein Condensate Trapped in a Harmonic Potential to the 1 D Problem, the 32th National Conference on Theoretical Physics, Da Nang, 2008.

[5] L. Bergé, Phys. Rep. 303 (1998) 259.

Received 15 August 2008. 